JOURNAL OF APPLIED CRYSTALLOGRAPHY

ISSN 1600-5767

Received 1 September 2021

Accepted 25 November 2021

Edited by S. Moggach, The University of Western Australia, Australia

Keywords: crystallographic education; teaching materials; four-circle X-ray diffractometers.

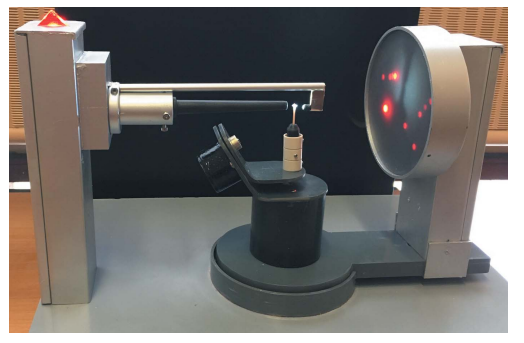

\section{CRISTAL-ITE: a single-crystal X-ray diffractometer scale model for scientific dissemination}

\author{
Michel Giorgi ${ }^{a, b *}$ and Yves Berchadsky ${ }^{c}$ \\ aAix Marseille Univ, CNRS, Spectropole, 52 avenue Escadrille Normandie Niemen, Marseille, 13013, France, \\ ${ }^{\mathbf{b}}$ Association de Cristallographie d'Aix-Marseille, Campus St Jérôme D11, 52 avenue Escadrille Normandie Niemen, \\ Marseille, 13013, France, and ' $A$ ix Marseille Univ, CNRS, ITE, 52 avenue Escadrille Normandie Niemen, Marseille, \\ 13013, France. *Correspondence e-mail: michel.giorgi@univ-amu.fr
}

This article presents the design and manufacture of an automated scale model of a four-circle single-crystal X-ray diffractometer that can be used for scientific dissemination. The purpose of this device is to reach out to the wider public and students to introduce them in an entertaining way to one of the laboratory apparatuses to which they do not usually have access, to talk to them about crystallography in the broadest sense, to develop concepts in various fields of science and technology, and to initiate interest and discussions. The main technical aspects of the project are described, with the expectation that such an approach could be useful to anyone involved in scientific dissemination and could be developed for other laboratory equipment and other disciplines. This kind of device can also be the subject of scientific and technological projects in close collaboration with educational institutions.

\section{Motivation}

The development of digital tools is an undeniable asset for teaching (Abdulrahaman et al., 2020), including crystallographic teaching (Kantardjieff, 2010; Ferrara et al., 2021). Remote learning, video clips, augmented reality and other educational devices offer almost unlimited perspectives to reach large numbers of people and interest them in science and technology, as the recent health situation has revealed (Pokhrel \& Chhetri, 2021). However, this period has also greatly emphasized the need for encounters, physical links and direct contact between people. Moreover, our own experience in disseminating knowledge as well as that of other colleagues elsewhere in the world regularly demonstrates that nonacademic teaching aids are very attractive means to talk about crystallography (and all sciences) to non-specialists. A lot of very interesting media have been developed in that sense, especially since 2014, International Year of Crystallography (https://www.iycr2014.org/learn/educational-materials), and include exhibitions, games, comics, workshops, crystal growing competitions and radio broadcasts (Orlov et al., 2006; GarciaRuiz et al., 2015; Hodeau \& Guinebretiere, 2015; Gražulis et al., 2015; Van Meervelt, 2017; Gratias \& Ravy, 2018; Casas, 2020). Scientific studies (Murphy et al., 2004; Weinberg et al., 2019) and testimonials (Zheng et al., 2018) also show that the wider public and school/college students are generally very enthusiastic about the idea of meeting laboratory staff, researchers and engineers, to discuss their research or more general scientific subjects.

With this in mind, we thought it would be interesting to 'take out' of the laboratory some 'mysterious' and complex equipment, bringing it to the attention of people who do not 
usually have the opportunity to see it - or even know that it exists - and use it as a basis for explaining various phenomena and concepts in an engaging way. Crystallography is a multidisciplinary field that links many areas of mathematics, science and engineering. Moreover, the various technologies used in the design and operation of a four-circle single-crystal X-ray diffractometer make it a very visual apparatus, likely to attract the eye and allowing one to explain many physical phenomena and mathematical concepts (electromagnetic radiation, diffraction, interference, Fourier transform) and to talk about crystallography in the broadest sense (state of matter, crystals, chemistry, biology etc.). Tabletop diffractometers exist but are still very heavy and out of financial reach for schools or scientific associations for educational projects. One can also find on the internet cheap assemblies to build an X-ray generator, but without the guarantee of sufficient safety for students or for use in a public space (Science Buddies Staff, 2021).

On behalf of the Association de Cristallographie d'AixMarseille (ACAM; http://acam.cristal-provence.fr) we have therefore chosen to design and create a scale model of this type of diffractometer, with the most common characteristics of the laboratory equipment, but safe, cheap and easily transportable to classrooms or public spaces.

\section{General overview}

For the technical specifications of the scale model, we focused on four main points: the most faithful reproduction possible of a real diffractometer but with a simplified design, the use of the lightest and least expensive materials possible, an easy programming of the operating parameters, and a high level of autonomy for use in all conditions.

Concerning the first point we were very much inspired by one of the superb videos created by Michel Evain for the teaching of crystallography, available with free access (https:// www.youtube.com/watch?v=_J34y87_EoA). The principles and the functioning of a four-circle single-crystal X-ray diffractometer are described, and the different movements are clearly modelled. The different points of view of the video also

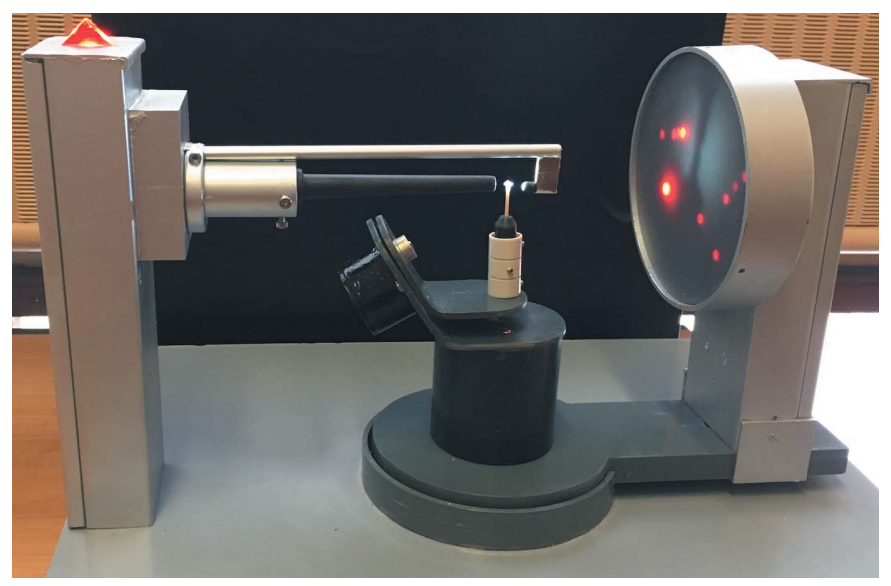

Figure 1

General overview of the scale model in operation. allowed us to imagine what the final object could look like in three dimensions. For the choice of materials, regarding the base of the model and most of its mobile or immobile pieces, we opted for polyvinyl chloride (PVC), of which the major part came from recycled resources easily found in laboratories, in workshops or at home. The motorizations of the movements are ensured by simple gear stepper motors with dedicated control cards that are soldered to a custom-made main electronic board together with an Arduino interface card for the control of the device. Finally, the diffraction spots are simulated by low-consumption LEDs. All these components can be easily found in electronics stores or on online sales sites and are very cheap. The most expensive items, relatively speaking, of the assembly are the Li-ion batteries with their charger, necessary to ensure a good autonomy of the system. That autonomy is reinforced by the use of the Arduino board, which enables the user to flash the control driver directly to the device. Finally, the base supporting the source, the goniometer and the detector was machined in a high-density PVC (HDPE) plate of $4 \mathrm{~mm}$ thickness to ensure sufficient rigidity without increasing the weight. The total size of the device is $40 \mathrm{~cm}$ wide, $37 \mathrm{~cm}$ deep and $30 \mathrm{~cm}$ high, and its weight without the optional plexiglass protective cover is $4.5 \mathrm{~kg}$ (Fig. 1).

\section{The mechanical parts}

\subsection{The goniometer}

For reasons of ease of design, we chose not to model the $\phi$ rotation, especially because of the difficulty of positioning the motor and the passage of its power cables. This movement being generally very small, we considered that this simplification could be justified.

The detector arm, the turret simulating the $\omega$ block and supporting the $\kappa$ and the $\phi$ blocks, and the $\kappa$ and $\phi$ blocks themselves were cut in PVC according to calculated dimensions in

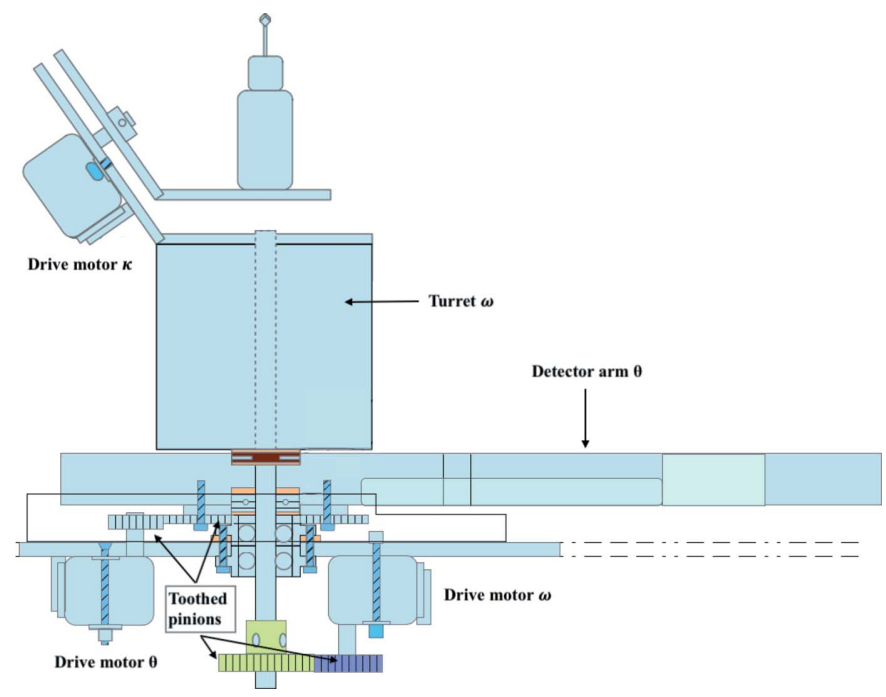

Figure 2

Scale drawing of the moving parts of the device and the motor assemblies with their associated toothed pinions. 
order to allow the crystal to remain at the centre of the beam and the goniometer rotations. The drive motor of the detector arm, $\theta$, is mounted under the main support plate, and its axis, mounted with a toothed pinion, crosses the plate to hook on a crown fixed in the arm and thus drive $\theta$ (Fig. 2). The motor of the movement $\omega$ is also fixed under the main plate next to the $\theta$ motor and transmits the movement through two toothed pinions, one of which is fixed on the lower part of the turret. Finally, the $\kappa$ plate is mounted directly on the axis of its own motor, which is itself fixed at the back of the support plate positioned at the top of the $\omega$ block.

As mentioned above, the rotation $\phi$ of the sample is not reproduced in the scale model. The sample holder is made from a $30 \mathrm{~mm}$ section of PVC pipe with a diameter of $16 \mathrm{~mm}$. Milled grooves and fixed hexagon screws represent the three centering elements that allow the sample to be adjusted on the real device (Fig. 1). A PVC rod of $1 \mathrm{~mm}$ diameter is used to hold a plastic faceted dummy crystal. The rod is removable and adjustable in height so that the operator can choose to adjust it in the beam and/or replace the fake sample by a real crystal. The sample holder has an adjustable height of about $50 \mathrm{~mm}$ overall.

\subsection{Simulation of the $X$-ray source}

The pseudo-X-ray source is made of $21 \mathrm{~cm}$ of PVC trunking. The cover is located at the back and allows access to the wiring of the various LEDs. It is topped by a transparent red Plexiglas prism in which a high-power LED is inserted to simulate the alert during the X-ray emission (Fig. 1). The collimator support is machined from a block of polyethylene, on which is fixed a double cylinder part in transparent polycarbonate, on which is fitted, fastened by three hexagon socket screws, a truncated cone part in HDPE. At the end of this piece there is a high-power white LED which, by lighting the crystal during the 'irradiation' phase, simulates the X-ray beam (obviously invisible in reality) for didactic reason. The well holder, which is supposed to protect the detector from the direct incident beam, is here made of an aluminium tube. It leads the wiring of an LED of the same nature as the collimator's and illuminates the sample in the same way on the other side at the time of 'irradiation' (Fig. 1).

\subsection{The detector and the simulation of the diffraction patterns}

The cylindrical part of the detector consists of a slice of HDPE tube with an external diameter of $125 \mathrm{~mm}$ and a cross section of $30 \mathrm{~mm}$. Two discs of $6 \mathrm{~mm}$ thickness obstruct this piece on both sides. The front part is perforated to accommodate red LEDs corresponding to three different predefined diffraction patterns (Fig. 3). The whole unit is fixed on a PVC trunking whose back cover is used to hide the wiring of the LEDs of the detector. The assembly can slide manually on the detector holder arm.

In order to simulate the diffraction pattern of a single crystal, three images were chosen from a laboratory experiment at different positions: low, medium and high resolution. High-intensity red LEDs are positioned in holes drilled in the front plate of the detector, and the LEDs that are common to each image are connected in parallel to illuminate at the same time. The relative intensity of each diffraction spot is simulated by the addition of resistors of values appropriate to each intensity level. Finally, the effect of diffusion on the pattern is represented by use of a translucent plate positioned as a screen on the detector (Fig. 1). The three images are synchronized to appear successively during the irradiation simulation carried out for the three positions of the goniometer previously defined and controlled by the system driver.

\section{Motorization, driver and system operation}

For the erasable programmable read-only memory (EPROM) board and the programming of the system, we have chosen the Arduino solution. Arduino is an open-source electronics platform based on easy-to-use hardware and software (https:// www.arduino.cc). The boards are able to read the input and turn it into an output. They are very affordable components,
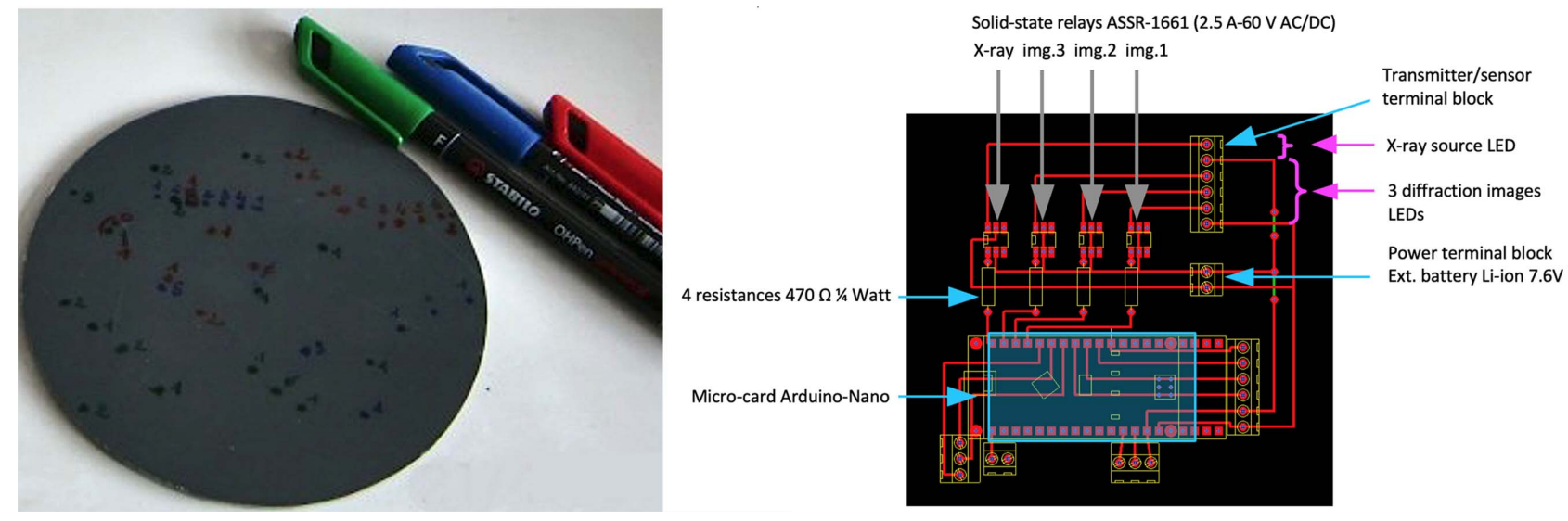

Figure 3

Left: marking of the LEDs' locations on the detector before drilling; each colour corresponds to one set of LEDs connected in parallel and lighting up at the same time. Right: electronic configuration of the three sets of LEDs (img.1-3) on the main board. 
and they can be chosen with different levels of complexity. We opted for the very simple Arduino-Nano board, which is sufficient to drive the unit. The Arduino language is an extremely simplified version of $\mathrm{C}$, and programming is made easier by many examples of all kinds that can be found online. The programming of CRISTAL-ITE has been realized from the integrated development environment (IDE) Arduino 1.8.10 under Windows 10 but was also tested on Mac OS X without problem. This IDE allows the programming of all Arduino-compatible boards and the transfer of programs to the EPROM.

The motorizations of the detector arm, the $\omega$ turret and the $\kappa$ plate are provided in three groups, each comprising a stepper motor with a gearbox and an Arduino-compatible control card. These sets allow the operator to control the repetitive movement and positioning of the three elements mentioned above, whose positions have been chosen beforehand in order to correspond to the low-, medium- and highresolution diffraction patterns. The three motor driver boards are mounted on the main board of CRISTAL-ITE, together with the Arduino-Nano board and several optoelectronics relays, necessary for the control of the lighting of the various LEDs. The whole system is located under the main base of the device and protected by a PVC plate (Fig. 4). A square hole, corresponding to the USB input of the Arduino-Nano board, was made in the front of the base, allowing the connection to a central unit for the transfer of the Arduino code. The system is powered by a pair of rechargeable Li-ion batteries $(2 \times$ 3.5 A h $)$ in series that provide $7.27 \mathrm{~V}(2 \times 3.635 \mathrm{~V})$. They are recharged through a suitable charger connected to a Jack socket placed at the rear of the system.

An ON/OFF switch placed at the back of the scale model main board allows the system to be powered up. The device can be switched on either with batteries only or with the

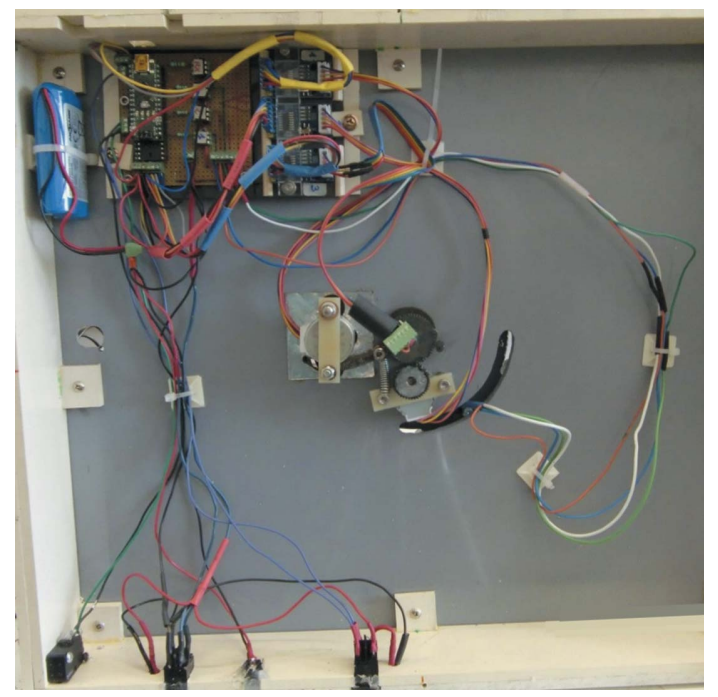

Figure 4

Bottom of the device, under the PVC base, showing the main electronic and mechanical elements. Top: the main board of CRISTAL-ITE with the Arduino components and its USB plug, and the Li-ion batteries (blue). Centre: the $\theta$ and $\omega$ arm motors. Bottom from left to right: reset button, main ON/OFF switch, Jack plug and pause switch. charger connected. Battery autonomy has been tested for a continuous operation of the device over a period of more than eight hours.

As soon as it is switched on, the CRISTAL-ITE Arduino board is powered up and starts its programming sequence. The system begins its automatic cycle with all the movements of the goniometer in its home position $(\theta, \omega$ and $\kappa$ are equal to $0^{\circ}$ ). All LEDs light up, including the first diffraction pattern, for $3 \mathrm{~s}$. Then the detector moves to $\theta=45^{\circ}$, followed by $\omega$ and $\kappa$, which rotate together to position themselves at pre-defined angles. The detector is illuminated with the second diffraction pattern and then $\theta$ moves to its last position, $90^{\circ}$, followed by $\omega$ and $\kappa$ for the simulation of the last irradiation at high resolution. Then the system repeats the operations in the opposite direction until it returns to the home position, and the sequence is repeated indefinitely until the unit is powered off by the main switch. For practical reasons of design and programming, switching the power back on does not allow the program to resume at the point of interruption but restarts a complete cycle. Therefore, the operator should switch off the system at the end of the cycle (detector facing the source) to avoid a possible shift in position when restarting. However, a repositioning of the different rotation blocks can also be done manually without problems, the stepper motors allowing such an operation. Finally, the user can interrupt the CRISTALITE program by simply pushing the PAUSE switch located at the back of the device. The interruption will take place during the next illumination of the detector and can be useful if the instructor wishes to spend time on pedagogical explanations (comments about diffraction, resolution, the Fourier transform...). Switching on again resumes the cycle. Finally, a reset button allows the operator to interrupt the program.

All scale model control parameters - time of LED exposure, delay between two sequences, positions of the goniometer - can be easily modified in the Arduino code and just need to be transferred to the EPROM board via the USB plug.

Virtual applications, like the EwaldSphere program which allows illustration of different aspects of the diffraction phenomenon (Barbour, 2018), can also be used in parallel with our model to complete the information given to the public and to go further into the development of some concepts.

\section{Conclusion}

We have developed an automated scale model of a four-circle single-crystal X-ray diffractometer. We believe that such a device, safe and easily transportable outside the laboratory, can be an attractive way to explain technological and scientific concepts to people who are not familiar with our research, with our laboratory devices and, more generally speaking, with our environment, which is often perceived as closed and mysterious. Crystallography lends itself particularly well to this type of approach, but all scientific disciplines can be presented in this way. Even more interesting and didactic, the design and construction of a laboratory apparatus could be the subject of educational projects in secondary schools through collaborative work: many examples show the benefits of such 
an approach for the acquisition of knowledge by students (Blumenfeld et al., 1991; Deria et al., 2017; Nalamalapu, 2017). Such a project would allow educators to approach, in an entertaining way, many technological and scientific notions, programming, and teamwork and could initiate real exchanges between students, teachers and scientists.

A video of the device in operation is available on the ACAM web site: http://acam.cristal-provence.fr/Notremaquette. Although exclusively in French for the moment, all the characteristics of our model as well as the design plans and the programming code can be obtained on request to the correspondence email.

\section{Funding information}

The following funding is acknowledged: Conseil Régional Provence-Alpes-Côte d'Azur (grant No. 2020_05727).

\section{References}

Abdulrahaman, M. D., Faruk, N., Oloyede, A. A., SurajudeenBakinde, N. T., Olawoyin, L. A., Mejabi, O. V., Imam-Fulani, Y. O., Fahm, A. O. \& Azeez, A. L. (2020). Heliyon, 6, e05312.

Barbour, L. J. (2018). J. Appl. Cryst. 51, 1734-1738.

Blumenfeld, P. C., Soloway, E., Marx, R. W., Krajcik, J. S., Guzdial, M. \& Palincsar, A. (1991). Educ. Psychol. 26, 369-398.

Casas, L. (2020). J. Appl. Cryst. 53, 1583-1592.

Deria, B., Yasar, O. \& Hasan, K. (2017). Res. Sci. Technol. Educ. 35, 131-148.
Ferrara, J. D., Cochran, A., Del Campo, M., Göb, C. R., Le Maguerès, P., Meyer, M., Puschmann, H., Schürmann, C., Stanley, A., Swepston, P. N., Tripathi, A., White, F. \& Wojciechowski, J. (2021). Struct. Dyn. 8, 010401.

Garcia-Ruiz, J. M., Otalora, F., Garcia-Caballero, A., GonzálezRamírez, L. A. \& Verdugo-Escamilla, C. (2015). J. Appl. Cryst. 48, 1264-1275.

Gratias, D. \& Ravy, S. (2018). France Inter, La Tête au Carré, France, https://www.franceinter.fr/emissions/la-tete-au-carre/la-tete-au-carre03-mai-2018.

Gražulis, S., Sarjeant, A. A., Moeck, P., Stone-Sundberg, J., Snyder, T. J., Kaminsky, W., Oliver, A. G., Stern, C. L., Dawe, L. N., Rychkov, D. A., Losev, E. A., Boldyreva, E. V., Tanski, J. M., Bernstein, J., Rabeh, W. M. \& Kantardjieff, K. A. (2015). J. Appl. Cryst. 48, 1964-1975.

Hodeau, J.-L. \& Guinebretiere, R. (2015). J. Appl. Cryst. 48, 12761289.

Kantardjieff, K. (2010). J. Appl. Cryst. 43, 1276-1282.

Murphy, C., Beggs, J., Carlisle, K. \& Greenwood, J. (2004). Int. J. Sci. Educ. 26, 1023-1035.

Nalamalapu, A. R. (2017). Int. J. Curr. Res. 9, 57492-57494.

Orlov, I., Schoeni, N. \& Chapuis, G. J. (2006). J. Appl. Cryst. 39, 595597.

Pokhrel, S. \& Chhetri, R. (2021). High. Educ. Future, 8, 133-141.

Science Buddies Staff (2021). How to Build an X-ray Machine, https:// www.sciencebuddies.org/science-fair-projects/project-ideas/Phys_p083/ physics/how-to-build-an-x-ray-machine.

Van Meervelt, L. (2017). Acta Cryst. A73, C510.

Weinberg, A. E., Sebald, A., Stevenson, C. A. \& Wakefield, W. (2019). Teach. Educ. 55, 190-213.

Zheng, S.-L., Chen, Y.-S., Wang, X., Hoffmann, C. \& Volkov, A. (2018). J. Appl. Cryst. 51, 909-914. 